\title{
Generalized Algorithms of Discrete Optimization and Their Power Engineering Applications
}

\author{
Roberto Berredo $^{1}$, Petr Ekel ${ }^{1,2}$, Helder Ferreira ${ }^{2,3}$, Reinaldo Palhares ${ }^{4}$, Douglas Penaforte ${ }^{5}$ \\ ${ }^{1}$ Advanced System Optimization Technologies-ASOTECH, Belo Horizonte, Brazil \\ ${ }^{2}$ Graduate Program in Electrical Engineering, Pontifical Catholic University of Minas Gerais, Belo Horizonte, \\ Brazil \\ ${ }^{3}$ CEMIG Distribution, Belo Horizonte, Brazil \\ ${ }^{4}$ Graduate Program in Electrical Engineering, Federal University of Minas Gerais, Belo Horizonte, Brazil \\ ${ }^{5}$ CEMIG Generation and Transmission, Belo Horizonte, Brazil \\ Email: rcberredo@gmail.com, pekel@superig.com.br, hlara@cemig.com.br, rpalhares@ufmg.br, \\ penaforte@cemig.com.br
}

Received 29 July 2015; accepted 23 August 2015; published 26 August 2015

Copyright (C) 2015 by authors and Scientific Research Publishing Inc.

This work is licensed under the Creative Commons Attribution International License (CC BY). http://creativecommons.org/licenses/by/4.0/

(c) (i) Open Access

\section{Abstract}

Generalized algorithms for solving problems of discrete, integer, and Boolean programming are discussed. These algorithms are associated with the method of normalized functions and are based on a combination of formal and heuristic procedures. This allows one to obtain quasioptimal solutions after a small number of steps, overcoming the NP-completeness of discrete optimization problems. Questions of constructing so-called "duplicate" algorithms are considered to improve the quality of discrete problem solutions. An approach to solving discrete problems with fuzzy coefficients in objective functions and constraints on the basis of modifying the generalized algorithms is considered. Questions of applying the generalized algorithms to solve multicriteria discrete problems are also discussed. The results of the paper are of a universal character and can be applied to the design, planning, operation, and control of systems and processes of different purposes. The results of the paper are already being used to solve power engineering problems.

\section{Keywords}

Discrete Optimization, Method of Normalized Functions, Duplicate Algorithms, Fuzzy Coefficients, Interrelated Models, Multiobjective Decision Making

\footnotetext{
${ }^{*}$ Corresponding author.
}

How to cite this paper: Berredo, R., Ekel, P., Ferreira, H., Palhares, R. and Penaforte, D. (2015) Generalized Algorithms of Discrete Optimization and Their Power Engineering Applications. Engineering, 7, 530-543. 


\section{Introduction}

Discrete, integer, and Boolean (in the general case, discrete) optimization problems have important applications in many fields [1] [2]. Taking this into account, it should be stressed that direct determination of discrete solutions to problems of discrete character is necessary. This is associated with the fact that even though at the cost of ignoring discreteness of parameters, with smoothing of functions, it is possible to replace an actual objective function by a convex or concave function defined on a convex region, with such an approach the danger exists that the objective function will be distorted (with a deviation from the optimum) or that the constraints will be violated (see a simple example in Section 3). Moreover, the transition from the discrete model to its convex analog can lead to considerable "coarsening" the model that often makes vapid its essence [3]. Finally, with orientation to discrete methods it is possible to pose and solve problems of combinatorial nature, which have previously not been considered [3].

Theoretical and experimental evaluations, discussed in [4] [5], for example, have revealed significant drawbacks of exact methods of discrete programming. Besides, estimates of computational complexity [6] in solving discrete problems indicate that their NP-completeness does not permit one to develop general methods with a polynomial dependence on the problem dimension [7]. Thus, the development and application of approximate methods are the main direction in the evolution of discrete programming.

The algorithms discussed in the present paper are based on a combination of formal (the method of normalized functions [8]) and heuristic (elements of the greedy heuristics [9]-[11], generally, providing the best heuristic among possible heuristics with a priori estimates) procedures. The algorithms permit one to obtain quasioptimal solutions after a small number of steps, overcoming the problem NP-completeness. They do not require an analytical specification of objective functions and constraints, ensuring the flexibility and the possibility to solve problems, for which adequate analytical descriptions are difficult or impossible.

In the process of formulating and solving a wide range of problems related to the design, planning, operation, and control of complex systems, one inevitably encounters different types of uncertainty [12]-[14]. Considering this, it should be stressed that taking into account the uncertainty factor in shaping mathematical models is to be inherent to the practice of systems analysis. This serves as a means for increasing the adequacy of the built models and, as a consequence, the credibility and factual effectiveness of solutions based on their analysis.

Investigations of recent years show the benefits of applying fuzzy set theory [15] to deal with various types of uncertainty. Its use in problems of optimization character offers advantages of both fundamental nature (the possibility of obtaining more effective, less "cautious" solutions) and computational character [16] [17]. Considering this, the present paper also reflects results related to modifying the generalized algorithms to solve discrete problems with fuzzy coefficients in objective functions and constraints as well as multiobjective discrete problems.

\section{Problem Formulation}

From the diversity of discrete optimization problems it is possible to distinguish two comprehensive classes. The first class is associated with the general problem of discrete programming, including integer, Boolean, and discrete programming problems proper. The problems with discrete variables may be reduced to integer or, in the general case, to Boolean models [1] [18]. However, such a reduction, usually, substantially, increases the problem dimensionality [3].

The second class of models is related to the problems of combinatorial type. In their solution, an extremum of the objective function is defined on a given finite discrete set $A$. The totality of objects obtained from $A$ (for example, combinations or permutations) as well as objects obtained as a result of executing logical operations on elements of $A$ [3] may be considered as a combinatorial space $D$. The problem is formulated as a search for $X^{0}=\left(x_{1}^{0}, \cdots, x_{n}^{0}\right)$ from $G \subseteq D$ providing an extremum of the objective function, i.e., $F(X) \rightarrow \underset{X \in G \in D}{\operatorname{extr}}$.

The combinatorial problems are the most difficult from the computational viewpoint. Their solution is based, in the main, on finiteness of $G \subseteq D$ and the problem specificity. Many combinatorial problems may be reduced to problems of integer or Boolean programming. For instance, Ibaraki [19] defines sufficient conditions of reducibility of combinatorial problems to integer programming models but shows that there is no a general algorithm for such reducibility, even it is realizable. Besides, in many cases reducibility is reached by accepting considerable assumptions, sharp increasing model dimension, and losing the possibility to effectively exploit com- 
binatorial properties of the initial problem [3].

In this connection, when solving discrete problems, it is important that their formulation and solution should exploit those properties and peculiarities of the problems, which promote their effective solution. Considering this, the desirability of allowing for constraints on the discreteness of in the form of discrete sequences

$$
x_{s_{i}}, \rho_{s_{i}}, \tau_{s_{i}}, \cdots, \quad s_{i}=1, \cdots, r_{i}
$$

has been validated in [18]; here $\rho_{s_{i}}, \tau_{s_{i}}, \cdots$ are technical, economic, etc. characteristics required for forming objective functions, constraints, and their increments, which correspond to the sth discrete (integer, Boolean) value of the variable $x_{i}$. Considering this, a maximization problem may be formulated as follows.

Assume we are given discrete sequences of the type (1) (increasing or decreasing, depending on the problem formulation). From them it is necessary to choose parameters that the objective

$$
\text { maximize } F\left(x_{s_{1}}, \rho_{s_{1}}, \tau_{s_{1}}, \cdots, \cdots, x_{s_{n}}, \rho_{s_{n}}, \tau_{s_{n}}, \cdots\right)
$$

is met while satisfying

$$
g_{j}\left(x_{s_{1}}, \rho_{s_{1}}, \tau_{s_{1}}, \cdots, \cdots, x_{s_{n}}, \rho_{s_{n}}, \tau_{s_{n}}, \cdots\right) \leq b_{j}, \quad j=1, \cdots, d .
$$

The objective function (2) is interpreted as concave and the constraints (3) are interpreted as convex.

Given the maximization problem (1)-(3), one can formulate a minimization problem. In particular, from the discrete sequences of the type (1) it is necessary to choose parameters that the objective

$$
\text { minimize } F\left(x_{s_{1}}, \rho_{s_{1}}, \tau_{s_{1}}, \cdots, \cdots, x_{s_{n}}, \rho_{s_{n}}, \tau_{s_{n}}, \cdots\right)
$$

is met while satisfying

$$
g_{j}\left(x_{s_{1}}, \rho_{s_{1}}, \tau_{s_{1}}, \cdots, \cdots, x_{s_{n}}, \rho_{s_{n}}, \tau_{s_{n}}, \cdots\right) \geq b_{j}, \quad j=1, \cdots, d .
$$

The objective function (4) is interpreted as convex and the constraints (5) are interpreted as concave.

\section{Solution Algorithms}

Let us consider the Boolean problem of maximization of

$$
F(x)=\sum_{i=1}^{n} c_{i} x_{i},
$$

while satisfying

$$
\sum_{i=1}^{n} a_{j i} x_{i} \leq b_{j}, \quad j=1, \cdots, d,
$$

where $c_{i}>0, i=1, \cdots, n, a_{j i}>0, \quad j=1, \cdots, d, i=1, \cdots, n$, and $b_{j}>0, \quad j=1, \cdots, d$.

The idea of one of popular methods, associated with the class of heuristic methods, may be illustrated by analyzing (6) and (7) for $d=1$ (the 0 - 1 knapsack problem). It is possible to assume that $x_{i}, i=1, \cdots, n$ are arranged as

$$
c_{1} / a_{1} \geq c_{2} / a_{2} \geq \cdots \geq c_{n} / a_{n} .
$$

It allows one to try to maximize (6) on the basis of the largest $c_{i} / a_{i}$, considering $x_{1}=1$, then $x_{2}=1$, and so on until (7) is observed. Similar methods are called greedy methods. Regardless of their "naivety", in many cases, as it was indicated above, they represent the best heuristic among other heuristics with a priory estimates. However, a specter of problems is not restricted by the case of $d=1$. Taking this into account, we discuss below ways of constructing algorithms for the general case $(d>1)$ to solve problems (linear as well as nonlinear), which can include not only Boolean, but also integer and discrete variables.

When analyzing (6) and (7) for $d=1$, the maximization is reached by expending only one type of resources. If $d>1$, the optimization process is stopped when a remaining amount of only one of resources is not sufficient for next incrementing any of $x_{i}, i=1, \cdots, n$. Thus, we can speak about "equivalence" of different types of resources from the point of view of terminating the process of maximizing (6). Therefore, it is advisable to have a single 
measure for different resources. This leads to the idea of normalization [8]. For example, the constraints (7) are reduced to a single arbitrary resource $b$ as

$$
a_{j i}^{(t)}=a_{j i} b / b_{j i}^{(t-1)}, \quad j=1, \cdots, d, \quad i=1, \cdots, n,
$$

where $t$ is the optimization step number.

Applying (9), it is possible to transform the constraints (7) to equal conditions. For instance, before the first optimization step we have

$$
\sum_{i=1}^{n} a_{j i}^{(0)} x_{i} \leq b, j=1, \cdots, d .
$$

Assume that at step $t$, variable $x_{i}$ is at its discrete level $x_{s_{i}}^{(t)}$ and its associated parameters $\rho_{i}, \tau_{i}, \cdots$ are at the respective levels $\rho_{s_{i}}^{(t)}, \tau_{s_{i}}^{(t)}, \ldots$. These can be gathered in what we introduce as the set $\xi_{s_{i}}^{(t)}=\left\{x_{s_{i}}^{(t)}, \rho_{s_{i}}^{(t)}, \tau_{s_{i}}^{(t)}, \cdots\right\}$. Then the algorithm of solving the maximization problem (1)-(3), taking into account the results of [3] [20], can be written in the following form.

1) The components of the vector $\left\{\Delta G_{i}^{(t)}\right\}$ are evaluated as follows:

$$
\Delta G_{i}^{(t)}=\max _{j} \Delta g_{j i}^{(t)}, \quad i \in I^{(t)}, j=1, \cdots, d,
$$

where $I^{(t)}$ is the set of variables at the th step which, at their present values satisfy all constraints (3). In (11), $\Delta g_{j i}^{(t)}$ is the increment in the $j$ th constraint when $\xi_{s_{i}}^{(t)}$ undergoes a step change from its level $s_{i}$ to the next level $s_{i}+1$ while all the other $\xi_{s_{k}}^{(t)}, k \neq i$ remain at their current levels $s_{k}$ :

$$
\Delta g_{j i}^{(t)}=\left[g_{j}\left(\xi_{s_{1}}^{(t)}, \cdots, \xi_{s_{i}+1}^{(t)}, \cdots, \xi_{s_{n}}^{(t)}\right)-g_{j}\left(\xi_{s_{1}}^{(t)}, \cdots, \xi_{s_{i}}^{(t)}, \cdots, \xi_{s_{n}}^{(t)}\right)\right] b / b_{j}^{(t-1)}, j=1, \cdots, d, i \in I^{(t)} .
$$

For $t=1$ we have $i \in I_{n} \quad\left(I_{n}\right.$ is the initial set of variables) and $b_{j}^{(t-1)}=b_{j}^{(0)}=b_{j}$.

2) If $I^{(t)}=\left\{i \mid \Delta G_{i}^{(t)} \leq b, i \in I^{(t)}\right\} \neq \varnothing$, then go operation 3 , otherwise the calculations are completed because the solution is obtained.

3) The components of the vector $\left\{\Delta F_{i}^{(t)}\right\}$ are calculated as follows:

$$
\Delta F_{i}^{(t)}=F\left(\xi_{s_{1}}^{(t)}, \cdots, \xi_{s_{i}+1}^{(t)}, \cdots, \xi_{s_{n}}^{(t)}\right)-F\left(\xi_{s_{1}}^{(t)}, \cdots, \xi_{s_{i}}^{(t)}, \cdots, \xi_{s_{n}}^{(t)}\right), \quad i \in I^{(t)} .
$$

4) If $I^{(t)}=\left\{i \mid \Delta F_{i}^{(t)}>0, i \in I^{(t)}\right\} \neq \varnothing$, then go to operation 5, otherwise the calculations are completed because the solution is obtained.

5) The components of the vector $\left\{V_{i}^{(t)}\right\}$ are calculated as:

$$
V_{i}^{(t)}=\Delta F_{i}^{(t)} / \Delta G_{i}^{(t)}, i \in I^{(t)} .
$$

6) The index $i=\theta_{t}$ of the variable to be incremented is determined from the following condition:

$$
V_{\theta_{t}}^{(t)}=\max _{i} V_{i}^{(t)}, \quad i \in I^{(t)} .
$$

7) We recalculate the current values of the following quantities:

$$
\begin{gathered}
x_{s_{i}}^{(t)}= \begin{cases}x_{s_{i}}^{(t)} & \text { if } i \neq \theta_{t}, i \in I^{(t)}, \\
x_{s_{i}+1}^{(t)} & \text { if } i=\theta_{t} ;\end{cases} \\
b_{j}^{(t)}=b_{j}^{(t-1)}-\Delta g_{j \theta_{t}}^{(t)} b_{j}^{(t-1)} / b, j=1, \cdots, d .
\end{gathered}
$$

8) If $I^{(t)}=\left\{i \mid s_{i}<r_{i}, i \in I^{(t)}\right\} \neq \varnothing$, then go to operation 1, taking $t=t+1$; otherwise the calculations are completed because the solution is obtained.

Commenting the algorithm of solving the maximization problem (1)-(3), it is necessary to indicate that the execution of its operation 1 provides determination of the constraint with the most scarce type of the resource $b_{j}^{(t)}, j=1, \cdots, d$ for every variable at the given step. In essence, the execution of (11) permits one to construct a convolution: $\sum_{i \in I^{(t)}} \Delta G_{i}^{(t)} \leq b$. Therefore, at each optimization step we obtain an increment of that $\theta_{t}$ th variable 
which maximizes the increment of the objective function per unit normalized resource $b$. In this connection, the utilization of (14) and (15) is similar to (8). Besides, the execution of operations 2 and 4 is directed at excluding variables that lead to violation of the constraints (3) or to decreasing the objective function of (2).

The problem of minimization (1), (4), and (5) is more difficult than the problem (1)-(3). In particular, in the case of maximization we stop changing the variable $x_{i}$ when at least one of the constraints (3) is violated. At the same time, in the minimization process the optimization is completed on any variable when all constraints (5) are obeyed. Therefore, there is in the case of maximization usually only one "deficient" constraint at each step requiring attention. At the same time, in minimization we have to pay attention to each constraint because the optimization process cannot be completed until all constraints (5) have been obeyed.

It is assumed that the initial constraints (5) are already normalized and are presented in the following form:

$$
g_{j}^{(0)}\left(\xi_{s_{1}}, \cdots, \xi_{s_{n}}\right)=g_{j}\left(\xi_{s_{1}}, \cdots, \xi_{s_{n}}\right) b / b_{j}^{(t-1)} \geq b, j=1, \cdots, d .
$$

The algorithm of solving the minimization problem (1), (4), and (5), using the results of [3] [20], can be presented in the following form.

1) The components of the vector $\left\{\Delta G_{i}^{(t)}\right\}$ are evaluated as follows:

$$
\Delta G_{i}^{(t)}=\sum_{j} \Delta g_{j i}^{(t)}, i \in I^{(t)}, j \in J^{(t)}
$$

In (19),

$$
\Delta g_{j i}^{(t)}=\left[g_{j}\left(\xi_{s_{1}}^{(t)}, \cdots, \xi_{s_{i}+1}^{(t)}, \cdots, \xi_{s_{n}}^{(t)}\right)-g_{j}\left(\xi_{s_{1}}^{(t)}, \cdots, \xi_{s_{i}}^{(t)}, \cdots, \xi_{s_{n}}^{(t)}\right)\right] b_{j}^{(t-1)} / b, \quad j \in J^{(t)}, i \in I^{(t)},
$$

where $J^{(t)}$ is the set of the constraints (5) at the th step. For $t=1$ we have $j \in J_{d}$ ( $J_{d}$ is the initial set of constraints); $i \in I_{n}$.

2) The components of the vector $\left\{\Delta F_{i}^{(t)}\right\}, i \in I^{(t)}$ are calculated in accordance with (13).

3) The components of the vector $\left\{V_{i}^{(t)}\right\}, \quad i \in I^{(t)}$ are calculated on the basis of (14).

4) The index $i=\theta_{t}$ of the variable to be incremented is determined from the following condition:

$$
V_{\theta_{t}}^{(t)}=\min _{i} V_{i}^{(t)}, \quad i \in I^{(t)} .
$$

5) We recalculate the current values of the quantities $x_{s_{i}}^{(t)}, \quad i \in I^{(t)}$, using (16), and

$$
b_{j}^{(t)}=b_{j}^{(t-1)}-\Delta g_{j l_{t}}^{(t)} \frac{b}{b_{j}^{(t-1)}}, \quad j \in J^{(t)} .
$$

6) If $J^{(t)}=\left\{j \mid b_{j}^{(t)}>0, j \in J^{(t)}\right\} \neq \varnothing$, then go to operation 7; otherwise the calculations are completed because the solution is obtained.

7) If $I^{(t)}=\left\{i \mid s_{i}<r_{i}, i \in I^{(t)}\right\} \neq \varnothing$, then go to operation 1, taking $t=t+1$; otherwise the calculations are completed because the problem has no solution.

Commenting the algorithm of solving the minimization problem (1), (4), and (5), it is necessary to indicate that the execution of its operation 1 provides the convolution of the set of constraints (5) at the given optimization step. In this connection, at each step of optimization we obtain an increment of that $\theta_{t}$ th variable which minimizes the increment of the objective function per unit total expenditure of normalized resources. The execution of operation 6 is associated with excluding such constraints (5) which are already satisfied. The algorithm has no operation similar to operation 4 of the algorithm of solving the maximization problem. This does not restrict a range of its applications because prior to using the algorithm it is possible to carry out minimization of the objective function (4) without considering the constraints (5).

Numerous comparisons of solutions for diverse types of problems given, for instance, in [1] [21], based on applying the algorithms presented above (with the upper bounds of the number of operations $N=(5 m n+6 m+11) n$ for Boolean and $N=(5 m n+6 m+11) \sum_{i=1}^{n} r_{i}$ for integer and discrete linear problems $)$ and exact methods, show their convincing agreement. This is also confirmed by numerous results on "good" behavior of the greedy algorithms [22] for wide classes of discrete optimization problems. However, the authors of [23] indicate that the 
greedy algorithms are to be used with caution. Taking into account these opinions, it is rational to have not only one, but several algorithms realizing different strategies. Considering this, so-called "duplicate” algorithms have been elaborated on the basis of a qualitative analysis of the problem statement. In particular, one of the "duplicate” algorithms is based on evaluating the components of the vector $\left\{\Delta G_{i}^{(t)}\right\}$ (operation 1 of the algorithm of solving the minimization problem) as follows:

$$
\Delta G_{i}^{(t)}=\min _{j} \Delta g_{j i}^{(t)}, i \in I^{(t)}, j \in J^{(t)},
$$

where $\Delta g_{j i}^{(t)}, i \in I^{(t)}, \quad j \in J^{(t)}$ are calculated on the basis of (12).

The utilization of the "duplicate" algorithms can be considered, in a certain measure, as a guarantee of obtaining optimal solutions. Furthermore, the analysis of one and the same problem on the basis of several algorithms allows obtaining a series of solutions of equal worth, which is important as well.

As an example of the use of the "duplicate" algorithm associated with applying (23) we consider a problem of selecting locations and sizes of capacitors for a distribution network whose scheme and parameters are shown in Figure 1. It also gives the loads of transformers as well as load flow. For simplicity, the problem is solved under a simplified statement directed at minimizing a total installed power of low voltage capacitors while the constraints on lower voltage limits for maximal load levels are satisfied.

The discrete sequence of low voltage capacitor sizes is the following:

$$
\begin{aligned}
& x_{1_{i}}, 0 \mathrm{kVAr} ; \\
& x_{2_{i}}, 78 \mathrm{kVAr} ; \\
& x_{3_{i}}, 156 \mathrm{kVAr} .
\end{aligned}
$$

It is necessary to select sizes of capacitors which minimize the objective function

$$
F=\sum_{i=1}^{i=5} x_{s_{i}}
$$

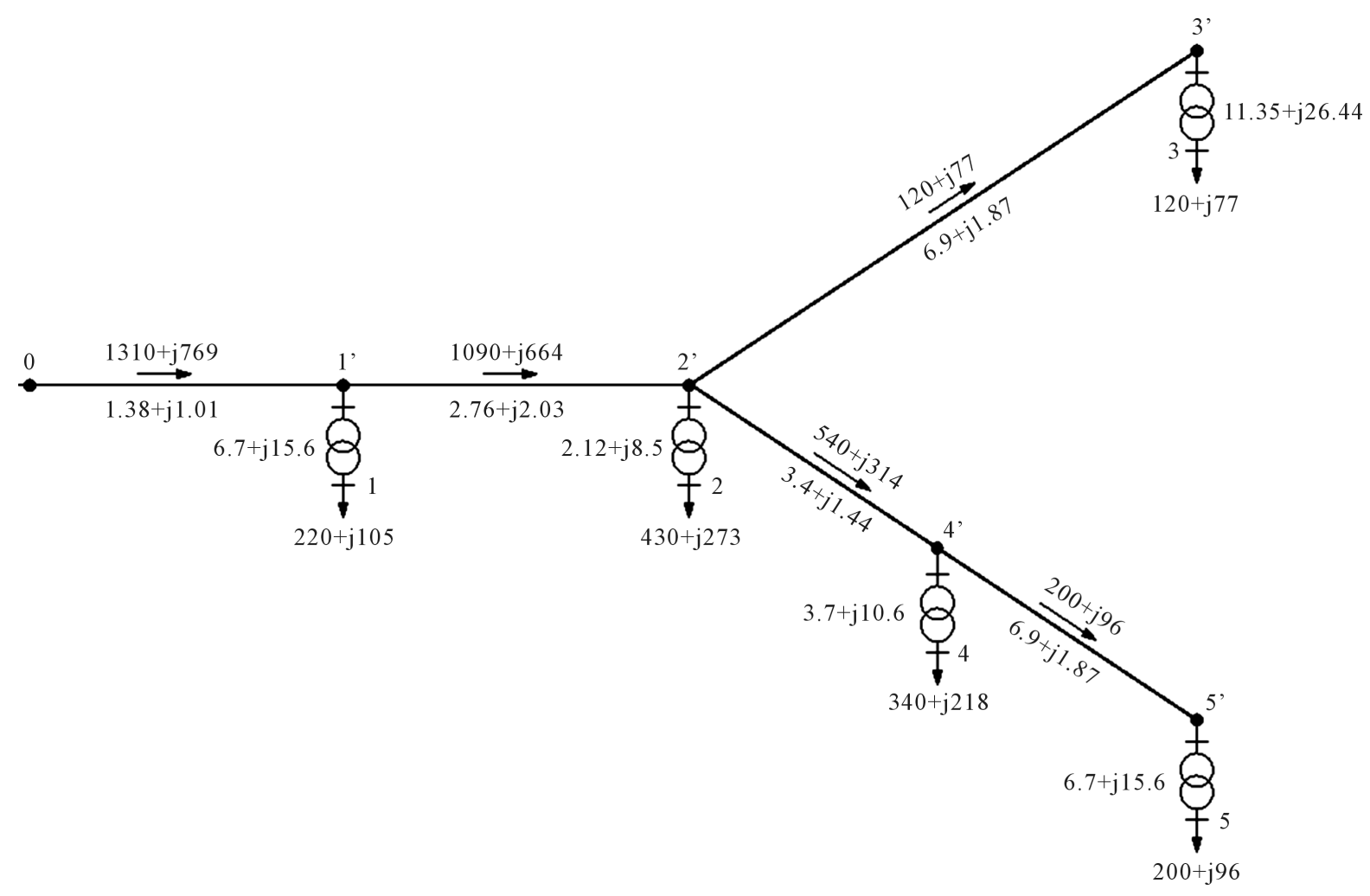

Figure 1. Network scheme, its parameters, and loads. 
while satisfying the following constraints on lower voltage limits:

$$
\begin{aligned}
& 1.01 x_{s_{1}}+11.54 x_{s_{2}}+3.04 x_{s_{3}}+3.04 x_{s_{4}}+3.04 x_{s_{5}} \geq 910 \\
& 1.01 x_{s_{1}}+3.04 x_{s_{2}}+31.35 x_{s_{3}}+3.04 x_{s_{4}}+3.04 x_{s_{5}} \geq 3550 \\
& 1.01 x_{s_{1}}+3.04 x_{s_{2}}+3.04 x_{s_{3}}+15.08 x_{s_{4}}+4.48 x_{s_{5}} \geq 840 \\
& 1.01 x_{s_{1}}+3.04 x_{s_{2}}+3.04 x_{s_{3}}+4.48 x_{s_{4}}+21.95 x_{s_{5}} \geq 1660
\end{aligned}
$$

The solution of (24)-(26), based on applying of the "duplicate" algorithm associated with the use of (23), is the following: $x_{1}=x_{1_{1}}=0 \mathrm{kVAr}, \quad x_{2}=x_{2_{2}}=78 \mathrm{kVAr}, \quad x_{3}=x_{3_{3}}=156 \mathrm{kVAr}, \quad x_{4}=x_{2_{4}}=0 \mathrm{kVAr}, \quad$ and $x_{5}=$ $x_{25}=78 \mathrm{kVAr}$. The corresponding value of the objective function (25) is $F=312 \mathrm{kVAr}$.

The solution of the problem (25) and (26) with ignoring the constraints (24) on variable discreteness, based on applying the simplex method of linear programming, is the following: $x_{1}=0 \mathrm{kVAr}, x_{2}=34 \mathrm{kVAr}, x_{3}=104$ $\mathrm{kVAr}, x_{4}=12 \mathrm{kVAr}$ and $x_{5}=54 \mathrm{kVAr}$. Rounding the obtained values to the nearest discrete ones does not permit one to obtain a feasible solution. Rounding the obtained values to the nearest higher discrete ones provides the following solution: $x_{1}=0 \mathrm{kVAr}, x_{2}=78 \mathrm{kVAr}, x_{3}=156 \mathrm{kVAr}, x_{4}=78 \mathrm{kVAr}$ and $x_{5}=78 \mathrm{kVAr}$. The corresponding value of the objective function (25) is $F=390 \mathrm{kVAr}$.

Another "duplicate" algorithm is associated with the results of [9] and is based on calculating $\Delta g_{j i}^{(t)}, i \in I^{(t)}$, $j \in J^{(t)}$ in the following form:

$$
\Delta g_{j i}^{(t)}=\min \left\{\left[g_{j}\left(\xi_{s_{1}}^{(t)}, \cdots, \xi_{s_{i}+1}^{(t)}, \cdots, \xi_{s_{n}}^{(t)}\right)-g_{j}\left(\xi_{s_{1}}^{(t)}, \cdots, \xi_{s_{i}}^{(t)}, \cdots, \xi_{s_{n}}^{(t)}\right), b_{j}^{(t-1)}\right]\right\}, j \in J^{(t)}
$$

with recalculating $b_{j}^{(t)}, \quad j \in J^{(t)}$ (operation 5 of the algorithm of solving the minimization problem) as

$$
b_{j}^{(t)}=b_{j}^{(t-1)}-\left[g_{j}\left(\xi_{s_{1}}^{(t)}, \cdots, \xi_{s_{i}+1}^{(t)}, \cdots, \xi_{s_{n}}^{(t)}\right)-g_{j}\left(\xi_{s_{1}}^{(t)}, \cdots, \xi_{s_{i}}^{(t)}, \cdots, \xi_{s_{n}}^{(t)}\right), b_{j}^{(t-1)}\right], \quad j \in J^{(t)} .
$$

As an additional means for possible improving the efficiency of solutions may serve the formulation and analysis of one and the same problem within the framework of mutually interrelated models (1)-(3) and (1), (4), and (5). Applying this approach, if we have the increasing (decreasing) sequences (1) for the problem (1)-(3), the sequences (1) are to be decreasing (increasing) for the problem (1), (4), and (5). Thus, it is possible to solve one and the same problem "from above" as well as "from below" as well. This approach is fruitful and also serves for solving problems with fuzzy coefficients discussed below.

The described results have a high degree of generality and have been used in solving diverse power engineering problems discussed below.

\section{Problems with Fuzzy Coefficients}

Although there are diverse formulations of optimization problems with fuzziness (for instance, [15] [24] [25], in opinion of the authors of [15] [24], the problems with fuzzy coefficients in objective functions and constraints are to be considered as a general class of fuzzy mathematical programming problems.

Generalizing (1)-(3), it is possible to construct the problem of choosing parameters from discrete sequences of the type (1) that the objective

$$
\operatorname{maximize} \tilde{F}\left(x_{s_{1}}, \rho_{s_{1}}, \tau_{s_{1}}, \cdots, \cdots, x_{s_{n}}, \rho_{s_{n}}, \tau_{s_{n}}, \cdots\right)
$$

is met while satisfying

$$
\tilde{g}_{j}\left(x_{s_{1}}, \rho_{s_{1}}, \tau_{s_{1}}, \cdots, \cdots, x_{s_{n}}, \rho_{s_{n}}, \tau_{s_{n}}, \cdots\right) \subseteq b_{j}, j=1, \cdots, m .
$$

The objective function of (29) and constraint (30) include fuzzy coefficients, as indicated by the $\sim$ symbol.

Generalizing (1), (4), and (5), it is possible to construct the problem of choosing parameters from discrete sequence of the type (1) that the objective

$$
\text { minimize } \tilde{F}\left(x_{s_{1}}, \rho_{s_{1}}, \tau_{s_{1}}, \cdots, \cdots, x_{s_{n}}, \rho_{s_{n}}, \tau_{s_{n}}, \cdots\right)
$$

is met while satisfying (30).

The models (1), (29), and (30) and (1), (31), and (30) generalize the models analyzed, for instance, in [26] [27] 
whose formulation with deterministic information have been considered in [3] [18].

An approach [17] [28] to handling the constraints such as (30) involves replacing each of them by a finite set of nonfuzzy (deterministic) constraints, represented in the form of inequalities. Depending on the essence of the problem, it is possible to change from the problem (1), (29), and (30) or (1), (31), and (30) to the problem (1), (29), and (3) or (1), (31), and (5) with fuzzy coefficients in the objective functions alone. The solution of the problem (1), (29), and (3) is possible on the basis of modifying the algorithm of solving the maximization problem (1)-(3). The solution of the problem (1), (31), and (5) is associated with modifying the algorithm of solving the minimization problem (1), (4), and (5) or the corresponding "duplicate" algorithms. In particular, the execution of algebraic operations on fuzzy numbers by means of the expressions (13) and (14) is accomplished on the basis of the results of [29].

To compare alternatives in accordance with (15) or (21) it is necessary to use the corresponding methods, considered, for instance, in [30]-[32]. Chen and Hwang [30] classify four groups of methods related to the ordering of fuzzy quantities. Among these groups, the authors of [33] consider the construction of fuzzy preference relations for pairwise comparisons as the most practical and justified way. Considering this, it is necessary to distinguish the fuzzy number ranking index introduced by Orlovsky [24] based on the conception of a membership function of a generalized preference relation.

If $\tilde{V}_{1}$ and $\tilde{V}_{2}$ have the membership functions $\mu\left(v_{1}\right)$ and $\mu\left(v_{2}\right)$, then quantity

$$
\eta\left\{\mu\left(v_{1}\right), \mu\left(v_{2}\right)\right\}=\sup _{\substack{v_{1}, v_{2} \in V \\ v_{1} \geq v_{2}}} \min \left\{\mu\left(v_{1}\right), \mu\left(v_{2}\right)\right\}
$$

is the degree of preference $\mu\left(v_{1}\right) \succeq \mu\left(v_{2}\right)$, while

$$
\eta\left\{\mu\left(v_{2}\right), \mu\left(v_{1}\right)\right\}=\sup _{\substack{v_{1}, v_{2} \in V \\ v_{1} \geq v_{2}}} \min \left\{\mu\left(v_{1}\right), \mu\left(v_{2}\right)\right\}
$$

is the degree of preference $\mu\left(v_{2}\right) \succeq \mu\left(v_{1}\right)$. These agree with several important fuzzy number ranking indices [17]. Examples of the application of the dependencies (32) and (33) are given in [17]. However, our experience shows that in many cases the membership functions of the alternatives $\mu\left(v_{1}\right)$ and $\mu\left(v_{2}\right)$ compared form flat apices (for example, [17]), i.e., they are so-called flat or trapezoidal fuzzy numbers [15]. In view of this, when using (32) and (30), it may happen that $\tilde{V}_{1}$ and $\tilde{V}_{2}$ are indistinguishable if $\eta\left\{\mu\left(v_{1}\right), \mu\left(v_{2}\right)\right\}=\eta\left\{\mu\left(v_{2}\right), \mu\left(v_{1}\right)\right\}$. In such situations the modified algorithms of discrete optimization do not allow one to obtain unique solutions: they "stop". This occurs also with other modifications of traditional mathematical programming methods (this is illustrated in [28] [34] by simple examples) because a combination of the uncertainty and the relative stability of optimal solutions produces so-called decision uncertainty regions. In this connection, other indices may be used as additional means for the ranking of fuzzy numbers.

The review of techniques which have been developed for ranking of fuzzy numbers can be found in [32]. This study covers the analysis of the fuzzy ranking indices proposed before 1998 (among more recent works in this field, it is possible to distinguish, for instance, [35] [36]).

The authors of [32] count more than 35 existing fuzzy number ranking indices, indicating that unlike in the case of real numbers, fuzzy quantities have no natural order. The idea with the ordering of fuzzy quantities is to convert a fuzzy quantity into a real number and base the comparison of fuzzy quantities on these real numbers. Each conversion way pays attention to a special aspect of fuzzy quantity. As a consequence, each approach suffers from some defects if only one real number is associated with each fuzzy quantity. Cheng [37] also indicates that many of indices produce different rankings for the same problem. The authors of [28] [31] [37] underline that fuzzy number ranking indices occasionally result in choices which appear inconsistent with intuition. Finally, the majority of indices for the ranking of fuzzy quantities have been proposed with the aspiration for obligatory distinguishing the alternatives. This is not natural because the uncertainty of information creates the decision uncertainty regions [17].

There is another approach that is better validated and natural for the practice of decision making. It is associated with the transition to multiattribute choosing alternatives in a fuzzy environment because the application of additional criteria (including the criteria of qualitative character, such as "investment attractiveness", "flexibility of development", etc.) can serve as a convincing means to contract the decision uncertainty regions.

Before starting to discuss questions of multiattribute decision making in a fuzzy environment, it is necessary 
to note that considerable contraction of the decision uncertainty regions may be obtained by formulating and solving one and the same problem within the framework of mutually interrelated models:

1) model of maximization (29) with the constraints (30) approximated by (3);

2) model of minimization (31) with the constraints (30) approximated by (5).

When using this approach, solutions dominated by the initial objective function are cut off from above as well as from below to the greatest degree [28]. It should be stressed that this approach is of a universal character and may be used in solving continuous problems as well.

Assume we are given a set $X$ of alternatives (from the decision uncertainty region), which are to be examined by $q$ criteria of quantitative and/or qualitative nature. This problem is presented by a pair $\langle X, R\rangle$ where $R=\left\{R_{1}, \cdots, R_{q}\right\}$ is a vector fuzzy preference relation [24] [28]. In this case, we have

$$
R_{p}=\left[X \times X, \mu_{R_{p}}\left(X_{k}, X_{l}\right)\right], p=1, \cdots, q, X_{k}, X_{l} \in X,
$$

where $\mu_{R_{\mathrm{p}}}\left(X_{k}, X_{l}\right)$ is a membership function of a fuzzy preference relation.

In (34), $R_{p}$ is defined as a fuzzy set of all pairs of $X \times X$, such that the membership function $\mu_{R_{p}}\left(X_{k}, X_{l}\right)$ represents the degree to which $X_{k}$ weakly dominates $X_{l}\left(X_{k}\right.$ is not worse than $\left.X_{l}\right)$ for the $p$ th criterion.

A convincing and natural approach to obtaining matrices $R_{p}$ is presented in [17] [28]. In particular, the availability of fuzzy or linguistic estimates of alternatives $F_{p}\left(X_{k}\right), \quad p=1,2, \cdots, q, \quad X_{k} \in X \quad$ (constructed on the basis of expert estimation or on the basis of aggregating information arriving from different sources of both formal and informal character) with the membership functions $\mu\left[F_{p}\left(X_{k}\right)\right], p=1,2, \cdots, q, \quad X_{k} \in X$ permits one, using the expressions (32) and (33), to construct $R_{p}, \quad p=1,2, \cdots, q$. Other our approaches to constructing matrices $R_{p}$ are discussed in [39] [40].

At the same time, fuzzy preference relations are not a unique form of preference representation. For instance, the authors of [41] [42] indicate eight formats which can be used to establish preferences among analyzed alternatives. It is natural that their application demands a conversion of all formats to a unique format which can be processed and analyzed. Considering the advantages and rationality of the application of fuzzy preference relations for this objective, the results of [17] [43] permit one to construct so-called transformation functions to convert different preference formats to fuzzy preference relations.

The basic procedures to deal with multiple criteria, when preferences are modeled as a vector $R$ of fuzzy preference relations, considered in [17], are the following: the construction of a set of nondominated alternatives on the basis of simultaneous considering all fuzzy preference relations [24] [28]; the lexicographic procedure bases on the step-by-step application of fuzzy preference relations for comparing alternatives [16] [28]; the construction of a set of nondominated alternatives on the basis of the intersection of sets of nondominated alternatives obtained for each fuzzy preference relation [20].

The indicated procedures have served for developing other techniques: the analysis of alternatives with fuzzy ordering of criteria [17] [24]; the analysis of alternatives based on the concept of fuzzy majority (based on the application of the so-called OWA operator, originally proposed in [44]) [17] [40]; the analysis of alternatives based on an outranking approach (by a means of a fuzzy version of the PROMETHEE [45]) [17].

\section{Multiobjective Problems with Discrete Variables}

When analyzing multiobjective models $(\langle X, F\rangle$ models [16] [17]), a vector of objective functions $F(X)=$ $\left\{F_{1}(X), \cdots, F_{q}(X)\right\}$ is considered, and the problem consists of optimizing all of them, i.e.,

$$
F_{p}(X) \rightarrow \underset{X \in L}{\operatorname{extr}}, \quad p=1, \cdots, q,
$$

where $L$ is a feasible region in $\mathbf{R}^{n}$.

The first step in solving the problem (32) is associated with determining a set of Pareto solutions $\Omega \subset L$ [46]. This step is useful; however it does not permit one to obtain unique solutions. It is necessary to choose a particular Pareto solution on the basis of information of a decision maker. There are three approaches to using this information [17] [47]: a priori, a posteriori, and adaptive.

When analyzing multiobjective problems, it is necessary to solve some questions related to normalizing objective functions, selecting principles of optimality, and considering priorities of objectives. Their solution and, therefore, development of multiobjective methods is carried out in the following directions [17] [48] [49]: scala- 
rization techniques, imposing constraints on objectives, utility function method, goal programming, and using the principle of guaranteed result. Without discussing these directions, it is necessary to point out that an important question in the multiobjective analysis is the solution quality. It is considered high if levels of satisfying objective functions are equal or close to each other (harmonious solutions) if we do not differentiate their importance [16] [50] (other directions may lead to solutions with high levels of satisfying some objectives that is reached by low levels of satisfying other objectives, for instance [17] [50]). From this point of view, it should be recorded the validity and advisability of the direction related to the principle of guaranteed result.

The lack of clarity in the concept of "optimal solution" is the basic methodological complexity in solving multiobjective problems. When applying the Bellman-Zadeh approach to decision making in a fuzzy environment [15] [51], this concept is defined with reasonable validity: the maximum degree of implementing goals serves as a criterion of optimality. This conforms to the principle of guaranteed result and provides constructive lines in obtaining harmonious solutions. The use of the Bellman-Zadeh approach permits one to realize an effective (from the computational standpoint) as well as rigorous (from the standpoint of obtaining solutions $X^{0} \in \Omega \subseteq L$ ) method of analyzing multicriteria models [16]. Finally, its use allows one to preserve a natural measure of uncertainty in decision making and to consider indices, criteria, and constraints of qualitative character.

When using the Bellman-Zadeh approach, objective functions $F_{p}(X), p=1, \cdots, q$ are replaced by fuzzy sets $A_{p}=\left\{X, \mu_{A p}(X)\right\}, X \in L, \quad p=1, \cdots, q$, where $\mu_{A p}(X)$ is the membership function of $A_{p}$. A fuzzy solution $D$ is defined as $D=\bigcap_{p=1}^{q} A_{p}$ with the membership function

$$
\mu_{D}(X)=\min _{1 \leq p \leq q} \mu_{A_{p}}(X), X \in L .
$$

The use of (36) allows one to get the solution

$$
\max \mu_{D}(X)=\max _{X \in L} \min _{1 \leq p \leq q} \mu_{A_{p}}(X)
$$

Therefore, the problem (35) is reduced to search for

$$
X^{0}=\arg \max _{X \in L} \min _{1 \leq p \leq q} \mu_{A_{p}}(X) .
$$

To obtain (38), one needs to build $\mu_{A_{p}}(X), \quad p=1, \cdots, q$, which reflect the capability of achieving own optima by $F_{p}(X), \quad X \in L, \quad p=1, \cdots, q$. This condition is satisfied if one chooses [17]:

$$
\mu_{A_{p}}(X)=\left[\frac{\max F_{p}(X)-F_{p}(X)}{\max _{X \in L} F_{p}(X)-\min _{X \in L} F_{p}(X)}\right]^{\lambda_{p}}
$$

for minimized objective functions or

$$
\mu_{A_{p}}(X)=\left[\frac{F_{p}(X)-\min _{X \in L} F_{p}(X)}{\max _{X \in L} F_{p}(X)-\min _{X \in L} F_{p}(X)}\right]^{\lambda_{p}}
$$

for maximized ones. In (39) and (40), $\lambda_{p}, \quad p=1, \cdots, q$ are importance factors for the corresponding objective functions.

The construction of (39) or (40) demands the solution of the following problems:

$$
\begin{aligned}
& F_{p}(X) \rightarrow \min _{X \in L}, \\
& F_{p}(X) \rightarrow \max _{X \in L} .
\end{aligned}
$$

Thus, the solution of the problem (35) on the basis of the Bellman-Zadeh approach demands analysis of $2 q+1$ monocriteria problems (41), (42), and (37), respectively.

Since $X^{0}$ is to belong to $\Omega \subseteq L$, it is necessary to build 


$$
\bar{\mu}_{D}(X)=\bigwedge_{p=1}^{q} \mu_{A_{p}}(X) \wedge \mu_{\pi}(X)=\min \left\{\min _{p=1, \cdots, q} \mu_{A_{p}}(X), \mu_{\pi}(X)\right\}
$$

where $\mu_{\pi}(X)= \begin{cases}1 & \text { if } X \in \Omega, \\ 0 & \text { if } X \notin \Omega \text {. }\end{cases}$

Procedures for solving the problem (37) discussed in [17] provide a line in obtaining $X^{0} \in \Omega \subseteq L$ in accordance with (43). Thus, it can be said about equivalence of $\bar{\mu}_{D}(X)$ and $\mu_{D}(X)$ and guarantees obtaining harmonious solutions which belong to the Pareto set. This eliminates the necessity of performing a cumbersome stage of constructing the Pareto set and opens up the real possibility of using the multicriteria approach in problems of the short-term planning, operation, and control.

Finally, the existence of additional conditions (indices, criteria or constraints) of qualitative character, defined by linguistic variables [15], reduces (38) to

$$
X^{0}=\arg \max _{X \in L} \min _{p=1, \cdots, q+s} \mu_{A_{p}}(X),
$$

where $\mu_{A_{p}}(X), X \in L, \quad p=q+1, \cdots, s$ are membership functions of fuzzy values of linguistic variables which reflect these additional conditions.

Taking the above into account, the solution of the multicriteria discrete problems is reduced to modifying the generalized algorithms of solving discrete optimization problems discussed above to solve the maxmin problem (38).

Although the results of the present Section do not take into account the uncertainty of initial information, they can be used within the framework of a general scheme of multicriteria analysis under information uncertainty [52] [53] with evaluating particular (monocriteria) and aggregated (multicriteria) risks in multiple scenarios.

\section{Power Engineering Applications}

The described results have found wide applications in the analysis of diverse problems of power engineering, related to improving reliability, quality, and economical feasibility of power supply. These problems are characterized by an extremely high number of variables and, often, by the impossibility of the adequate analytical description of objective functions and constraints.

The following classes of problems of power systems and subsystems have been solved with the use of the results of the present paper:

- choice and allocation of means for increasing reliability of power supply in distribution systems in different settings;

- reinforcement (allocation of reactive power sources, allocation of voltage regulators, and reconduction) of distribution systems in different settings;

- real-time active power control in power systems and subsystems;

- real-time voltage and reactive power control in distribution systems in different settings.

As an example, it possible to present the solution of the problem of allocating reactive power sources in distribution systems [54].

Traditionally, problems of reactive power compensation in distribution systems are associated with the selection of locations, sizes, and types of capacitors to minimize the objective function of an economical nature, while the constraints on upper and lower voltage limits at different load levels are satisfied. However, our experience in solving the problems of reactive power compensation shows that the necessity to simultaneously observe constraints on upper and lower voltage limits at different buses creates essential obstacles. It is not uncommon to face situations when these constraints induce empty feasible regions. These obstacles can be avoided by minimizing the objective function of an economical nature as well as the objective function which reflects a volume of poor quality energy consumption. Besides, if the problem is associated with the determination of capacitor types (fixed or switched), the quantity of objectives should be increased.

Taking this into account, the developed computing platform EPODIAN [54] includes tools to solve reactive power compensation problems in monocriteria as well as in multicriteria settings for large-scale distribution networks.

Table 1 demonstrates the results of the application of EPODIAN for the allocation of reactive power sources in a distribution network 13.8/0.22 kV of one of substations of the Energy Company of Minas Gerais (CEMIG). 
Table 1. Solution results.

\begin{tabular}{cccc}
\hline Solution & $\begin{array}{c}\text { Economic objective } \\
\text { function (BR\$· } 10^{3} \text { ) }\end{array}$ & $\begin{array}{c}\text { Poor quality energy } \\
\text { consumption (MWh/year) }\end{array}$ & $\begin{array}{c}\text { Total installed reactive } \\
\text { power (kVAr) }\end{array}$ \\
\hline$I$ & 708 & 826 & 600 \\
$A$ & 543 & 472 & 630 \\
$B$ & 661 & 384 & 1215 \\
$C$ & 568 & 396 & 900 \\
\hline
\end{tabular}

This network includes 2 feeders with more than 5000 consumers connected to them. The total length of feeders is $729 \mathrm{~km}$. They are modeled by 9660 electrical nodes. In Table $1, I$ is an initial state, $A$ is a monocriteria solution minimizing the objective function of the economical nature, $B$ is a monocriteria solution minimizing the objective function which reflects a volume of poor quality energy consumption, and $C$ is a multicriteria solution providing a compromise between the solutions $A$ and $B$. All solutions were obtained less than $30 \mathrm{~s}$ on a conventional dual-core desktop PC.

\section{Conclusions}

In this paper, the generalized algorithms for solving problems of discrete, integer, and Boolean programming are discussed. These algorithms are based on a combination of formal procedures (associated with the method of normalized functions) and informal procedures (related to elements of greedy heuristics). The application of the algorithms allows one to obtain quasi-optimal solutions after a small number of steps, thus overcoming the NPcompleteness of discrete optimization problems. Questions of constructing so-called "duplicate" algorithms are considered. Their use as well as the formulation and solution of one and the same discrete optimization problem within the framework of mutually related models serve as means for possible improving the quality of discrete problem solutions.

The approach to solving optimization problems, formalized within the framework of "soft" models containing fuzzy coefficients in objective functions and constraints, has been discussed. This approach is associated with modifying traditional mathematical programming methods and, in particular, the generalized algorithms presented in the paper. It is based on solving one and the same problem within the framework of mutually related models to maximally cut off dominated alternatives from above as well as from below. The subsequent contraction of the decision uncertainty regions is associated with reducing the problem to multiattribute choosing alternatives in a fuzzy environment.

It has shown the possibility of rational solving multiobjective discrete problems on the basis of applying the Bellman-Zadeh approach to decision making in a fuzzy environment and modifying the generalized algorithms presented in the paper.

The results of the paper have found wide applications in the analysis of power engineering problems, related to improving the reliability, quality, and economic efficiency of power supply.

\section{Acknowledgements}

This research was supported by the National Council for Scientific and Technological Development of Brazil (CNPq) - grants 305036/2011-4, 307466/2011-6, and the Energy Company of Minas Gerais (CEMIG)—R\&D ANEEL Program projects GT480 and D535.

\section{References}

[1] Taha, H.A. (1982) Operations Research: An Introduction. 3rd Edition, Macmillan Publishing, New York.

[2] Brown, D.E. and White, C.C. (1990) Operations Research and Artificial Intelligence: The Integration of ProblemSolving Strategies. Kluwer Academic Publishers, London. http://dx.doi.org/10.1007/978-94-009-2203-7

[3] Ekel, P.Ya. (1990) Models and Methods of Discrete Optimization of Power Supply Systems. KPI, Kiev. (In Russian)

[4] Jeroslow, R.C. (1974) Trivial Integer Programs Unsolvable by Branch-and-Bound. Mathematical Programming, 6, 105-109. http://dx.doi.org/10.1007/BF01580225 
[5] Korbut, A.A. and Finkelshtein, Yu.Yu. (1984) Approximate Methods of Discrete Programming. Soviet Journal of Computer and System Sciences, 22, 155-167.

[6] Garey, M.A. and Johnson, D.S. (1979) Computers and Intractability: A Guide to the Theory of NP-Completeness. W.H. Freeman, New York.

[7] Papadimitrou, C.H. and Steiglitz, K. (1982) Combinatorial Optimization: Algorithms and Complexity. Prentice-Hall, Englewood Cliffs.

[8] Berzin, E.A. (1974) Optimal Resource Allocation and Elements of System Synthesis. Russkoe Radio, Moscow. (In Russian)

[9] Dobson, G. (1982) Worst-Case Analysis of Greedy Heuristics for Integer Programming with Nonnegative Data. Mathematics of Operations Research, 7, 515-531. http://dx.doi.org/10.1287/moor.7.4.515

[10] Syslo, M., Deo, N., and Kowalik, J.S. (1983) Discrete Optimization Algorithms with Pascal Programs. Prentice-Hall, Englewood Cliffs.

[11] Cormen, T.H., Leiserson, C.E., Rivest, R.L. and Stein, C. (2009) Introduction to Algorithms. 3rd Edition, MIT Press, Cambridge, MA.

[12] French, S. (1995) Uncertainty and Imprecision: Modelling and Analysis. Journal of the Operational Research Society, 7, 70-79. http://dx.doi.org/10.1057/jors.1995.8

[13] Zimmermann, H. (2000) An Application-Oriented View of Modeling Uncertainty. European Journal of Operational Research, 122, 190-198. http://dx.doi.org/10.1016/S0377-2217(99)00228-3

[14] Antunes, C.H. and Dias, C.L. (2007) Editorial: Managing Uncertainty in Decision Support Models. European Journal of Operational Research, 181, 1425-1426. http://dx.doi.org/10.1016/j.ejor.2006.03.049

[15] Pedrycz, W. and Gomide, F. (1998) An Introduction to Fuzzy Sets: Analysis and Design. MIT Press, Cambridge, MA.

[16] Ekel, P.Ya. (2202) Fuzzy Sets and Models of Decision Making. Computers and Mathematics with Applications, 44, 863-875. http://dx.doi.org/10.1016/S0898-1221(02)00199-2

[17] Pedrycz, W., Ekel, P. and Parreiras, R. (2011) Fuzzy Multicriteria Decision-Making: Models, Methods, and Applications. John Wiley \& Sons, Chichester.

[18] Zorin, V.V. and Ekel, P.Ya. (1980) Discrete-Optimization Methods for Electrical Supply Systems. Power Engineering, 18, 19-30.

[19] Ibaraki, T. (1976) Integer Programming Formulation of Combinatorial Optimization. Discrete Mathematics, 16, 39-42. http://dx.doi.org/10.1016/0012-365X(76)90091-1

[20] Ekel, P.Ya. and Neto, F.H.S. (2006) Algorithms of Discrete Optimization and Their Applications to Problems with Fuzzy Coefficients. Information Sciences, 176, 2846-2868. http://dx.doi.org/10.1016/j.ins.2005.06.001

[21] Hu, T.C. (1969) Integer Programming and Network Flows. Addison-Wesley, New York.

[22] Ausiello, G., Crescenzi, P., Gambosi, G., Kan, V., Marchetti-Spaccamela, A. and Protasi, M. (1999) Complexity and Approximation. Springer, Berlin. http://dx.doi.org/10.1007/978-3-642-58412-1

[23] Band-Jensen, J., Gutin, G. and Yeo, A. (2004) When the Greedy Algorithm Fails. Discrete Optimization, 1, $121-127$. http://dx.doi.org/10.1016/j.disopt.2004.03.007

[24] Orlovsky, S.A. (1981) Problems of Decision Making with Fuzzy Information. Nauka, Moscow. (in Russian)

[25] Delgado, M. (1994) Fuzzy Optimization: Recent Advances. Physica-Verlag, Heidelberg.

[26] Herrera, F. and Verdegay, J.L. (1995) Three Models of Fuzzy Integer Linear Programming. European Journal of Operations Research, 83, 581-593. http://dx.doi.org/10.1016/0377-2217(93)E0338-X

[27] Herrera, F. and Verdegay, J.L. (1996) Fuzzy Boolean Programming Problems with Fuzzy Costs: A General Study. Fuzzy Sets and Systems, 81, 57-76. http://dx.doi.org/10.1016/0165-0114(94)00324-6

[28] Ekel, P., Pedrycz, W. and Schinzinger, R. (1998) A General Approach to Solving a Wide Class of Fuzzy Optimization Problems. Fuzzy Sets and Systems, 97, 49-66. http://dx.doi.org/10.1016/S0165-0114(96)00334-X

[29] Dubois, D. and Prade, H. (1979) Fuzzy Real Algebra: Some Results. Fuzzy Sets and Systems, 2, 327-348. http://dx.doi.org/10.1016/0165-0114(79)90005-8

[30] Chen, S.J. and Hwang, C.L. (1992) Fuzzy Multiple Attribute Decision Making: Methods and Applications. Springer, New York. http://dx.doi.org/10.1007/978-3-642-46768-4

[31] Lee-Kwang, H. (1999) A Method for Ranking Fuzzy Numbers and Its Application to Decision-Making. IEEE Transactions on Fuzzy Systems, 7, 677-685. http://dx.doi.org/10.1109/91.811235

[32] Wang, K. and Kerre, E.E. (2001) Reasonable Properties for the Ordering of Fuzzy Quantities (I). Fuzzy Sets and Systems, 118, 375-385. http://dx.doi.org/10.1016/S0165-0114(99)00062-7 
[33] Horiuchi, K. and Tamura, N. (1998) VSOP Fuzzy Numbers and Their Fuzzy Ordering. Fuzzy Sets and Systems, 93, 197-210. http://dx.doi.org/10.1016/S0165-0114(96)00206-0

[34] Galperin, E.A. and Ekel, P.Ya. (2005) Synthetic Realization Approach to Fuzzy Global Optimization via Gamma Algorithm. Mathematical and Computer Modelling, 41, 1457-1468. http://dx.doi.org/10.1016/j.mcm.2004.02.039

[35] Abbasbandy, S. and Hajjari, T. (2009) A New Approach for Ranking of Trapezoidal Fuzzy Numbers. Computers and Mathematics with Applications, 57, 413-419. http://dx.doi.org/10.1016/j.camwa.2008.10.090

[36] Sadi-Nezhad, S. and Shahnazari-Shahrezaei, P. (2013) Ranking Fuzzy Numbers Using Preference Ratio: A Utility Function Approach. Decision Science Letters, 2, 149-162. http://dx.doi.org/10.5267/j.dsl.2013.03.002

[37] Cheng, C.H. (1998) A New Approach for Ranking Fuzzy Numbers by Distance Methods. Fuzzy Sets and Systems, 95, 307-313. http://dx.doi.org/10.1016/s0165-0114(96)00272-2

[38] Fodor, J. and Roubens, M. (1994) Fuzzy Preference Modelling and Multicriteria Decision Support. Kluwer Academic Publishers, Boston. http://dx.doi.org/10.1007/978-94-017-1648-2

[39] Parreiras, R. and Ekel, P. (2013) Construction of Nonreciprocal Fuzzy Preference Relations with the Use of Preference Functions. Pesquisa Operacional, 33, 305-323. http://dx.doi.org/10.1590/S0101-74382013000200010

[40] Kokshenev, I., Parreiras, R.O., Ekel, P.Ya., Alves, G.B. and Menicucci, S.V. (2015) A Web-Based Decision Support Center for Electrical Energy Companies. IEEE Transactions on Fuzzy Systems, 23, 16-28. http://dx.doi.org/10.1109/TFUZZ.2014.2312984

[41] Zhang, Q., Chena, J.C.H. and Chong, P.P. (2004) Decision Consolidation: Criteria Weight Determination Using Multiple Preference Formats. Decision Support Systems, 38, 247-258. http://dx.doi.org/10.1016/S0167-9236(03)00094-0

[42] Zhang, Q., Wang, Y. and Yang, Y. (2007) Fuzzy Multiple Attribute Decision Making with Eight Types Of Preference Information. Proceedings of 2007 IEEE Symposium on Computational Intelligence in Multicriteria Decision Making, Honolulu, 1-5 April 2007, 288-293. http://dx.doi.org/10.1109/MCDM.2007.369103

[43] Herrera-Viedma, E., Herrera, F. and Chiclana, F. (2002) A Consensus Model for Multiperson Decision Making with Different Preferences Structures. IEEE Transactions on Systems, Man and Cybernetics, 32, 394-402. http://dx.doi.org/10.1109/TSMCA.2002.802821

[44] Yager, R.R. (1988) On Ordered Weighted Averaging Aggregation Operators in Multi-Criteria Decision Making. IEEE Transactions on Systems, Man and Cybernetics, 18, 183-190. http://dx.doi.org/10.1109/21.87068

[45] Li, W.X. and Li, B.Y. (2010) An Extension of the Promethee II Method Based on Generalized Fuzzy Numbers. Expert Systems with Applications, 37, 5314-5319. http://dx.doi.org/10.1016/j.eswa.2010.01.004

[46] Pareto, V. (1886) Cours D’économie Politique. Lousanne Rouge, Lousanne.

[47] Coelho, C.A.C. (2002) Evolutionary Multi-Objective Optimization: Critical Review. In: Sarker, R., Mohammadian, M. and Yao, X., Eds., Evolutionary Optimization, Kluwer Academic Publishers, Boston, 117-146.

[48] Miettinen, K.M. (1999) Nonlinear Multiobjective Optimization. Kluwer Academic Publishers, Boston.

[49] Ehrgott, M. (2005) Multicriteria Optimization. Springer-Verlag, Berlin.

[50] Ekel, P.Ya. and Galperin, E.A. (2003) Box-Triangular Multiobjective Linear Programs for Resource Allocation with Application to Load Management and Energy Market Problems. Mathematical and Computer Modelling, 37, 1-17. http://dx.doi.org/10.1016/S0895-7177(03)80001-8

[51] Bellman, R.E. and Zadeh, L.A. (1970) Decision-Making in a Fuzzy Environment. Management Science, 17, $141-164$. http://dx.doi.org/10.1287/mnsc.17.4.B141

[52] Ekel, P.Ya., Martini, J.S.C. and Palhares, R.M. (2008) Multicriteria Analysis in Decision Making Under Information Uncertainty. Applied Mathematics and Computation, 200, 501-516. http://dx.doi.org/10.1016/j.amc.2007.11.024

[53] Pereira Jr., J.G., Ekel, P.Ya., Palhares, R.M. and Parreiras, R.O. (2015) On Multicriteria Decision Making under Conditions of Uncertainty. Information Sciences, 234, 44-59. http://dx.doi.org/10.1016/j.ins.2015.06.013

[54] Araujo, W.J., Ekel, P.Ya., Filho, R.P.F., Kokshenev, I.V. and Schuffner, H.S. (2011) Monocriteria and Multicriteria Based Placement of Reactive Power Sources in Distribution Systems. International Journal of Applied Mathematics and Informatics, 5, 240-248. 